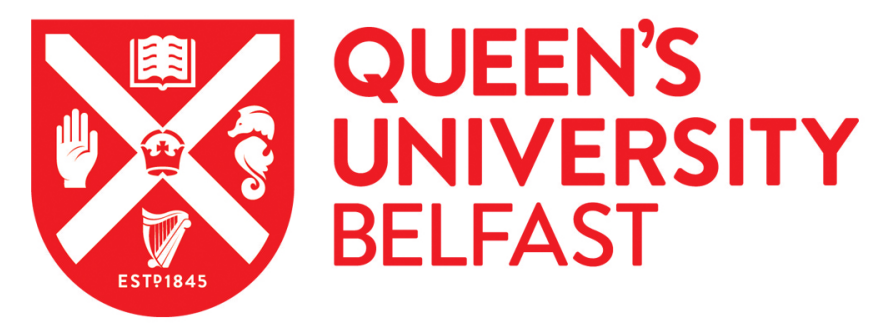

\title{
Three-dimensional cultures of normal human osteoblasts: proliferation and differentiation potential in vitro and upon ectopic implantation in
} nude mice.

Dickson, G., Ferrera, D., Poggi, S., Biassoni, C., Astigiano, S., \& Barbieri, O. (2002). Three-dimensional cultures of normal human osteoblasts: proliferation and differentiation potential in vitro and upon ectopic implantation in nude mice. Bone, 30(5)(5), 718-725. https://doi.org/10.1016/S8756-3282(02)00691-9

Published in:

Bone

Queen's University Belfast - Research Portal:

Link to publication record in Queen's University Belfast Research Portal

\section{General rights}

Copyright for the publications made accessible via the Queen's University Belfast Research Portal is retained by the author(s) and / or other copyright owners and it is a condition of accessing these publications that users recognise and abide by the legal requirements associated with these rights.

Take down policy

The Research Portal is Queen's institutional repository that provides access to Queen's research output. Every effort has been made to ensure that content in the Research Portal does not infringe any person's rights, or applicable UK laws. If you discover content in the Research Portal that you believe breaches copyright or violates any law, please contact openaccess@qub.ac.uk. 


\title{
Three-dimensional Cultures of Normal Human Osteoblasts: Proliferation and Differentiation Potential In Vitro and Upon Ectopic Implantation in Nude Mice
}

\author{
D. FERRERA, ${ }^{1}$ S. POGGI,${ }^{1}$ C. BIASSONI,${ }^{1}$ G. R. DICKSON,${ }^{2}$ S. ASTIGIANO ${ }^{3}$ O. BARBIERI, ${ }^{1}$ \\ A. FAVRE, ${ }^{4}$ A. T. FRANZI, ${ }^{5}$ A. STRANGIO,${ }^{5}$ A. FEDERICI,${ }^{6}$ and P. MANDUCA ${ }^{1}$ \\ ${ }^{1}$ Dipartimento di Oncologia, Biologia e Genetica, Università di Genova, Genova, Italy \\ ${ }^{2}$ Anatomy Department, Trauma Research Group, Queen's University, Belfast, Ireland \\ ${ }^{3}$ Istituto per la Ricerca sul Cancro, Genova, Italy \\ ${ }^{4}$ Istituto G. Gaslini, Genova, Italy \\ ${ }^{5}$ Sezione di Anatomia, Di. Me. S., Università di Genova, Genova, Italy \\ ${ }^{6}$ Ospedale di Recco, Genova, Italy
}

We report the establishment in vitro of three-dimensional (3D) cultures of human osteoblasts (hOB) derived from normal adults and supported uniquely by the extracellular matrix (ECM) they deposit. Osteoblasts were cultured in 3D cultures in vitro for up to 120 days. The $3 D$ cultures, examined at 25, 31, and 48 days, expressed protein markers of osteoblastic cells, namely osteonectin, collagen type I, fibronectin, osteopontin, bone sialoprotein, biglycan, and decorin. Sequentially, alkaline phosphatase (AP) and then Ca incorporation, mineralization of matrix (monitored by histochemistry and transmission electron microscopy), and finally osteocalcin expression, were detected in the 3D cultures. Ultrastructurally, morphology progressed from early to mature osteoblast and to osteocyte-like. Cells were embedded in a matrix with organized collagen type I fibers containing, increasingly with time of culture, needle-shaped crystals, often associated with matrix vesicles, characteristic of those in bone. During the culture (up to 120 days) there was an outgrowth of proliferating osteogenic cells from the 3D structure. Subcutaneous implantation in nude mice for 20 days of osteoblasts cultured in 3D culture for different lengths of time in vitro, showed progression of mineralization from the inner region of the implant outward, with peripheral cells being embedded in nonmineralized, collagen-rich matrix. The 3D implants were invaded by vessels derived from the host. (Bone 30:718-725; 2002) (C) 2002 by Elsevier Science Inc. All rights reserved.

Key Words: Human osteoblasts; Three-dimensional (3D) cultures; In vitro osteogenic differentiation; Progression of osteogenesis in vivo.

Address for correspondence and reprints: Dr. Paola Manduca, Dipartimento di Oncologia, Biologia e Genetica, Università di Genova, C. Europa 26, Genova 16132, Italy. E-mail: man-via@unige.it

D. F. and S. P. contributed to this investigation equally.

\section{Introduction}

Studies of osteoblastic cells from a number of animal species in two-dimensional (2D) cultures in vitro have revealed a general pattern of phenotypic progression during osteogenesis, $, 2,29,35$ establishing a hierarchy of determination events from a common mesenchymal precursor and identifying the key regulator genes involved in osteogenic determination. ${ }^{3,23,28,33}$ Also, cell-cell interactions via gap junctions, ${ }^{6,9}$ cell shape, and cytoskeletal organization, dependent on integrin-mediated interaction with specific components of the endogenous extracellular matrix (ECM), ${ }^{27,31}$ have been shown to determine cell metabolism, gene expression and differentiation, and cellular response to stimuli. ${ }^{17}$

Osteoblast survival and apoptosis ${ }^{4,19}$ depend on endogenous growth factors, and often associated with the ECM. Progression of cell differentiation and/or the maturation of the osteogenic ECM require developmentally regulated expression of endogenous metalloproteinases ${ }^{11}$ (Manduca et al., unpublished data).

Full osteogenic differentiation, up to the deposition of mineral in the ECM, can be resumed in vitro in 2D cultures from osteoblasts from fetal and young animals and, to a certain extent, in those derived from children (prepubertal), whereas adultderived (postpubertal) human osteoblasts in culture usually cannot complete osteogenesis up to mineralization. This finding has limited the study of events of the late phase of osteogenic progression in human cells and made problematic some of their potential applications for both basic and applied studies. It has long been debated as to whether the behavior of adult-derived osteoblasts is due to an intrinsic lack of osteogenic and/or proliferation potentials of these cells or if this block could be overcome by changing the culture conditions.

Three dimensional cultures of osteoblasts derived from rodents and from osteosarcomas were obtained and these underwent full osteogenic differentiation. These were based on the utilization of supports, as gels and sponges of collagen type I, PLGA foam, DegraPol, agarose, Matrigel, or methylcellulose. . $^{10,15,16,18,20,22,25,26,30,32,34,37,39}$ Normal human osteogenic cells, osteoblasts from prepubertal-age donors, or bone marrow stromal fibroblasts adhering to scaffolding of HAP/ TC, ${ }^{16,20,25,26,30,34}$ collagraft, ${ }^{26}$ collagen sponges, ${ }^{15,39}$ or bone 
chips $^{5}$ were utilized to produce specifically 3D structures designed for direct implantation in vivo.

Only recently there was a report that addition of transforming growth factor (TGF)- $\beta$ to monolayer cultures of adult human osteoblasts induces the formation of 3D structures, which, in the presence of the growth factor, progresses to mineralization, ${ }^{24}$ suggesting the relevance of 3D culture in maintaining the osteogenic potential of human osteoblastic cells. The investigators did not examine the role of exogenous growth factor in the progression of osteogenic differentiation.

Our first aim was to obtain an in vitro culture model for postpubertal human osteoblasts that would progress to full differentiation in vitro. We sought to assess in vitro the late molecular events of differentiation and investigate the role of cell-cell and cell-ECM relationships in osteogenic progression. The method established involves mechanical folding of monolayer cultures.

We show that maintenance of the self-produced microenvironment in osteoblast 3D cultures is sufficient to preserve osteogenic competence and to affect the overall maturation of the ECM deposed and organized by human adult-derived osteoblasts. Indeed, in this endogenous microenvironment, concomitant proliferation and full osteogenic differentiation took place. This was monitored by the expression of a panel of specific osteogenic markers, through the detection of type I collagen organization in fibers and, late in the culture, detection of mineral aggregates in the ECM by transmission electron microscopy (TEM). All this demonstrated osteoblast progression to the late phase of osteogenesis in 3D cultures, and the fact that the osteoblast endogenous microenvironment was sufficient to achieve full differentiation in vitro.

Our second aim was to establish whether the 3D culture could be transposed successfully to an in vivo microenvironment and if differentiation continued therein. We therefore implanted 3D cultures that had progressed to different stages of differentiation in vitro under the skin of nude mice. There, these $3 \mathrm{D}$ cultures became vascularized throughout and progressed to mineralization, suggesting that they represent a good model for in vivo osteogenic grafts to utilize with the aim of studying the effects of drugs and other systemic factors on adult human osteoblasts in selected phases of the osteogenic progression.

\section{Materials and Methods}

\section{Cells and 3D Cultures In Vitro}

Cells were obtained from trabecular fragments from long bones of women and men who were 25-73 years of age, free of bone-related disease, and not taking corticoids or medications for bone metabolism. Bone dissected free from soft tissue, after washing extensively in phosphate-buffered saline (PBS), was minced into fragments of about $1 \mathrm{~mm}^{3}$ and enzymatically digested with two changes of collagenase type IA $(260 \mathrm{U} / \mathrm{mL})$, as previously described. ${ }^{14,29}$ The bone fragments were incubated in a $5 \% \mathrm{CO}_{2}$ humidified incubator at $37^{\circ} \mathrm{C}$ in Coon's modified $\mathrm{F} 12$ medium, prepared omitting $\mathrm{Ca}^{2+}$, supplemented with $5 \mu \mathrm{g} / \mathrm{mL}$ each of insulin, transferrin, and $\mathrm{Na}$ selenite, with $50 \mathrm{IU} / \mathrm{mL}$ penicillin, $50 \mu \mathrm{g} / \mathrm{mL}$ streptomycin, and $20 \%$ of fetal calf serum (Seromed, Berlin, Germany). The cells proliferating out of the chips were trypsinized and passaged at a 1:2 dilution in medium containing $2 \mathrm{mmol} / \mathrm{L} \mathrm{Ca}$. Confluent cultures with three to five passages in 35-mm-diameter Petri dishes were set in medium supplemented with $100 \mu \mathrm{g} / \mathrm{mL}$ of ascorbic acid and $10 \mathrm{mmol} / \mathrm{L}$ $\beta$-glycerophosphate (differentiation medium). After 9 days (unless stated otherwise), the monolayer was mechanically dislodged as a whole sheet with a plastic micropipette and then transferred for $16 \mathrm{~h}$ to a differentiation medium in a Falcon tube. At this point, it collapsed randomly in a relatively compact 3D structure, which was then collected with tweezers and set for continuation of culture in a Petri dish, which was submerged in differentiation medium. Throughout the culture, medium was changed every second day. The 3D cultures were obtained from ten different individuals.

\section{Dosage of Alkaline Phosphatase and Calcium}

The 3D cultures were rinsed in PBS and collected in $1 \mathrm{~mL}$ of $0.1 \%$ sodium dodecylsulfate (SDS), mechanically disrupted by homogenization. AP was measured with a P-5656 Kit (Sigma $\mathrm{Co}$ ) and calcium was assessed with a $587 \mathrm{Kit}$ (Sigma). Both were normalized to DNA content that was determined by fluorimetry after Hoechst staining. All determinations were done in duplicate and data are expressed as medians. Deviation was 5\% and was not indicated.

\section{Preparation of Samples for Analysis by Histochemistry and Immunohistochemistry}

The 3D cultures were fixed in $4 \%$ paraformaldehyde and $2 \%$, sucrose, dehydrated, and embedded in paraffin. Sections were obtained from 3D cultures derived from osteoblasts taken from least two different individual donors in vitro, and from at least four different individual donors in vivo, were utilized for analysis of each timepoint with the experimental approaches indicated below. Sections were stained with hematoxylin-eosin (Merck \& Co., Inc., Whitehouse Station, NJ), Van Gieson $(0.1 \%$ acid fucsin in picric acid, $0.25 \% \mathrm{HCl}$ ), Sigma-Aldrich, Milan, Italy, and Von Kossa. Alkaline phosphatase was detected with an 86-C Kit (Sigma).

Immunofluorescence was done on sequential sections of $3 D$ cultures in vitro. Antisera

(Table 1) were diluted in PBS and $0.2 \%$ heat-inactivated bovine serum albumin (BSA). Dilutions of the specific antiserum, time of incubation at $4{ }^{\circ} \mathrm{C}(3 \mathrm{~h})$, concentration and time of incubation $(2 \mathrm{~h})$ with secondary antibody (goat anti-rabbit Immunoglobulin G [IgG], Jackson Immunoresearch, West Grove, PA), photographic exposure, and development times were the same for all samples. Two different 3D cultures were processed at each timepoint in vitro.

\section{Preparation of Samples for TEM}

The 3D cultures were washed in PBS before fixation at $4^{\circ} \mathrm{C}$ using $3 \%$ gluteraldehyde in $0.1 \mathrm{~mol} / \mathrm{L}$ sodium cacodylate buffer $(\mathrm{pH}$ 7.2-7.4) for $6 \mathrm{~h}$ to overnight, buffer washed in sodium cacodylate buffer, and then postfixed in $1 \%$ osmium tetroxide in $0.1 \mathrm{~mol} / \mathrm{L}$ sodium cacodylate buffer for $2 \mathrm{~h}$ (all from Scientific Ltd. UK). Dehydration was undertaken in an ascending series of ethanol $(70 \%, 90 \%$, and $100 \%)$ prior to immersion in propylene oxide, followed by impregnation and embedding in Spurr's resin (TAAB Labs, UK) using a standard procedure. ${ }^{38}$ Ultrathin sections were prepared using an ultramicrotome (Reichert OMU2), with a Drukker diamond knife (DCM Medical, Limerick, Ireland), lifted onto copper grids, and stained with uranylacetate and Reynolds lead citrate. Images of cells and ECM of cultured ossicles were recorded onto Kodak fine-grain electron-image film using a transmission electron microscope (Jeol 100CXII) and with electron micrographs produced on multigrade photographic paper (IIford). 
Table 1. Antisera utilized for immunodetection of osteoblast protein markers in three dimensional cultures

\begin{tabular}{lll}
\hline Antiserum & \multicolumn{1}{c}{ Specificity } & \multicolumn{1}{c}{ Source/reference } \\
\hline Osteocalcin & Bovine, cross-reacting to human & LF-32 \\
Bone sialoprotein & Human & LF-6 $\left({ }^{12}\right)$ \\
Osteopontin & Human & LF-7 $\left({ }^{12}\right)$ \\
Biglycan & Human & LF-15 $\left({ }^{13}\right)$ \\
Decorin & Human & LF-30 $\left({ }^{13}\right)$ \\
Osteonectin & Human & LF \\
Collagen I & Human & Pasteur, France \\
Fibronectin & Human & Dako, USA \\
Goat IgG FITC & Rabbit & Jackson Immunoresearch, USA
\end{tabular}

All primary antibodies were developed in the rabbit antisera obtained from Dr. L. Fisher, NIH, Bethesda, MD.

KEY: FITC, fluorescein isothiocyanate; IgG, immunoglobulin G; LF,

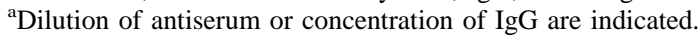

\section{Animals, Implants, and Explants}

Immunodeficient 6-10-week-old female nude mice (Harlan, Udine, Italy) were used as subcutaneous transplant recipients. Operations were performed in accordance with protocols approved by the internal ethics committee of the Advanced Biotechnology Center, Genoa, Italy, under anesthesia (obtained by intraperitoneal injection of $2.5 \%$ Avertin [Aldrich Chemical Co., Milwaukee, WI]). One subcutaneous pocket was produced by blunt dissection on each dorsal side of the mouse, wherein a single 3D culture was placed. Incisions were closed with surgical suture. Twenty days later the 3D cultures were explanted, fixed, included, sectioned, and analyzed.

Chemicals used were from Sigma Co. unless noted otherwise.

\section{Results}

Alkaline Phosphatase Expression and Calcium Incorporation During Osteogenesis in $2 D$ and $3 D$ Cultures In Vitro

The 3D structures were obtained from osteoblasts derived from ten different adult donors (of either gender, age range 25-73 years) after monolayer culturing in differentiating conditions for 9 days. At this timepoint osteoblasts had deposited a refractive matrix and expressed low levels of alkaline phosphatase (AP), which, if the culture is maintained as a monolayer, would peak in at week 3 of differentiation and decline thereafter. In 3D cultures AP was expressed in a manner similar to that of 2D cultures, both in time sequence and with regard to enzyme levels, whereas $\mathrm{Ca}$ incorporation in 3D cultures usually reached levels about tenfold higher than in 2D cultures. (Figure 1 shows a representative results from two experiments, a culture derived from a 25 -yearold woman and another from a 69-year-old man, respectively.)

Cells outgrew the dish within few days of 3D culture, and formed a monolayer adhering to the culture dish that, within 1.5-2 weeks, filled the 35-mm-diameter culture dish (not shown). We calculated that a 3D structure made of $8-9 \times 10^{4}$ cells, and whose DNA content remained unchanged over 35 days of culture, would generate an outgrowth of about $5 \times 10^{5}-10^{6}$ cells in 120 days. These cells became positive for AP upon reaching confluence (not shown). Osteogenic cells continued to outgrow 3D structures upon transfer to new dishes, for at least four sequential transfers and 120 days.

The size of the $3 \mathrm{D}$ cultures themselves increased during the culture period. This increase was due mostly to the apposition of the ECM, with the relative increase of the ratio between the ECM and cells being clearly visible upon comparison of $a$ vs. $b$ in Figure 2A. The amount of total proteins in the 3D structures shows a continual increase with time of culture, up to 3.5 -fold, whereas DNA level remains steady (measured up to 35 days, data not shown).

Analysis of Expression of Osteogenic Markers In Vitro by Histochemistry and Immunohistochemistry

The 3D cultures prepared from monolayers after 9 days in differentiation conditions were analyzed after total culture times of 25,31 , and 48 days, respectively. At the three timepoints, sequential sections of the $3 \mathrm{D}$ structures were examined by immunofluorescence with specific antibodies for the expression of characteristic osteogenic proteins, by histochemistry for AP expression, and for mineralization by Von Kossa stain (Figure 2). Figure 2 shows immunodetection in 3D structures analyzed after 25 and 48 days in culture and of histochemistry after 25,31 , and

A

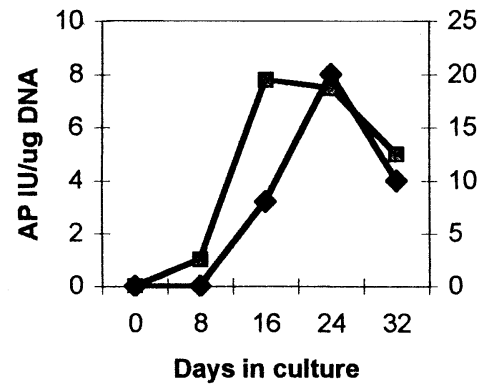

B

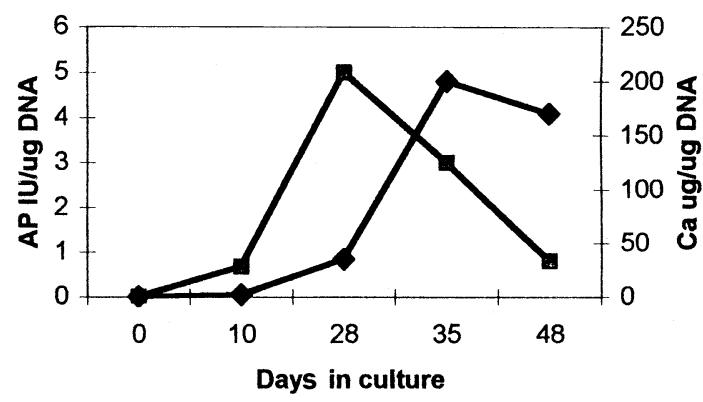

Figure 1. Alkaline phosphatase expression and $\mathrm{Ca}$ incorporation in $3 \mathrm{D}$ osteoblasts cultured in vitro. Time-regulated expression of AP and gradual increase with time of insoluble calcium incorporation during osteogenesis in 2D (A) and 3D (B) cultures. 
A

$\mathbf{B}$
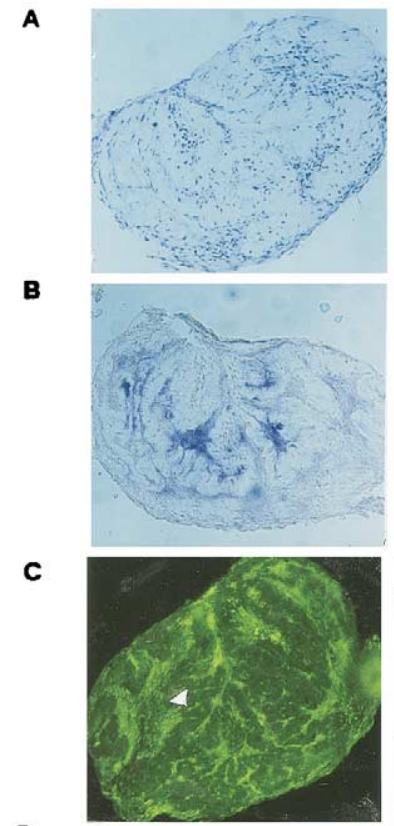

D
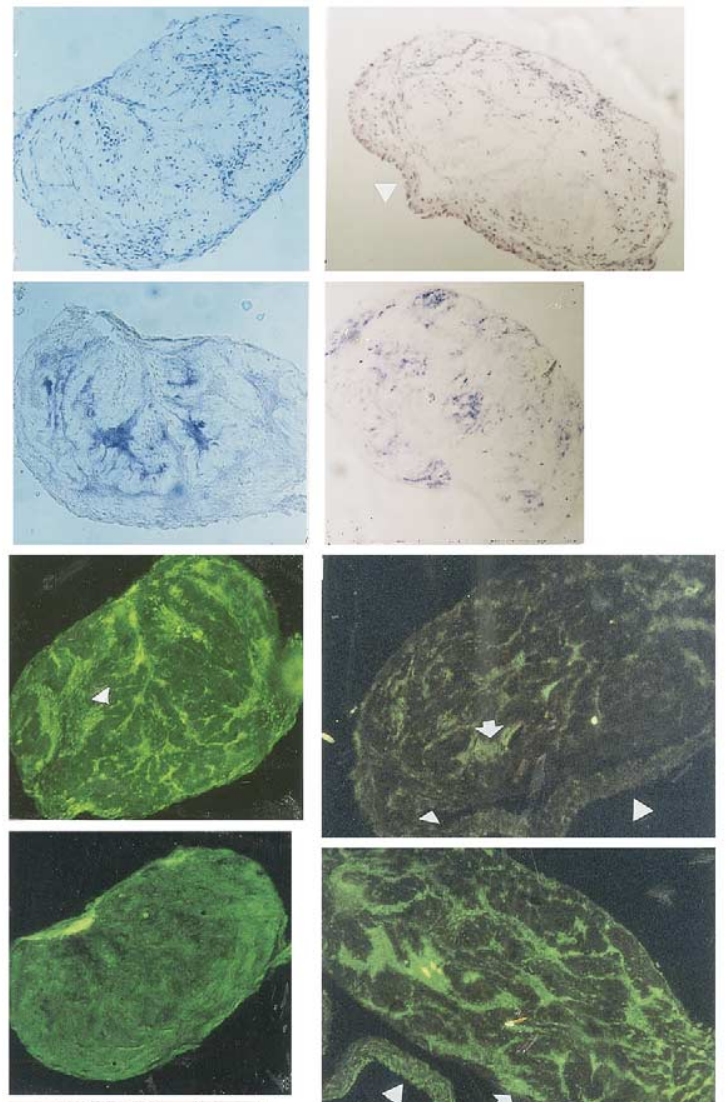

E
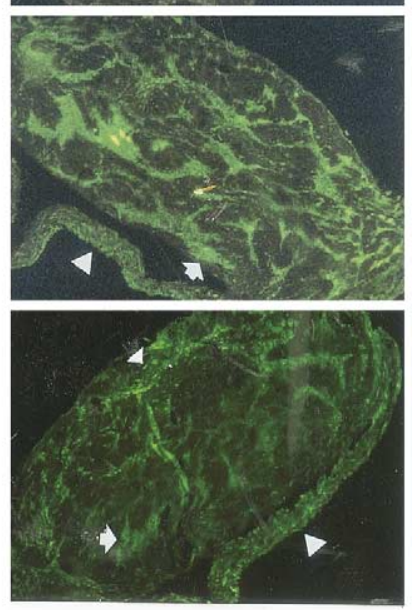

Figure 2. Histochemistry and immunohistochemistry of 3D cultures after various durations of culture time in vitro. Sections from 3D cultures in vitro were: (A) stained with Hematoxylin-eosin, after (a) 25 days and (b) 48 days of culture in differentiation conditions; (B) tested for alkaline phosphatase, after (a) 25 days and (b) 31 days of culture in differentiation conditions; (C) decorated by immunofluorescence with antiserum against osteonectin, after (a) 25 days and (b) 48 days of culture in differentiation conditions; (D) decorated by immunofluorescence with antiserum against osteopontin, after (a) 25 days and (b) 48 days of culture in differentiation conditions; and (E) stained with (a) Von Kossa after 31 days or decorated by (b) immunofluorescence with antiserum against osteocalcin after 48 days of culture in differentiation conditions. Small arrow heads: immunofluorescence-positive cells; arrows: ECM regions. Large arrowheads point to the layer of cells growing out from 3D structures, examined at 48 days. Original magnification: $\times 150$; (b) in (A) is $\times 100$.

48 days in culture. Expression of osteonectin, osteopontin, and osteocalcin are illustrated, but bone sialoprotein, decorin, biglycan, collagen type I, and fibronectin were also investigated. All these proteins were detected in sections of 3D cultures analyzed at 25 and 48 days. Biglycan expression was tested and detected only at 25 days (not shown). Osteocalcin was not detected when tested at 25 days (not shown), but was present at 48 days. Confluent cells outgrowing the Petri dish from the 3D structure tested at 48 days, and included in the samples analyzed, were also positive for all these osteogenic markers. ECM staining was also detected for all proteins and proteoglycans tested. Immunodetection for each marker was done in the same experiment and under identical conditions, on serial sections of 3D structures at each timepoint, and thus the suggestion emerged that some of these markers are modulated according to time in culture. For instance, a decreased intensity was noted for osteonectin (and fibronectin), whereas more intense staining was evident for decorin (not shown) at the longest timepoint of 3D cultures. Accumulation of proteins in the matrix was most evident for osteopontin. AP was expressed at 25 and 31 days but was undetectable at 48 days (not shown). This is consistent with the timing of its decline in $2 \mathrm{D}$ cultures, with the quantity of enzyme measured in the 3D structures, and with loss of AP expression in osteocytes in vivo. Von Kossa staining was negative at 25 days (not shown), only slightly positive at 31 days, and positive in at 48 days (not shown), consistent with increasing mineral deposition (see next subsection) and with the quantity of $\mathrm{Ca}$ associated in insoluble form with the ECM, as shown in Figure 1 (top) Taken together these data demonstrate the characteristic osteogenic phenotype of the cells and the progression of marker expression and maturation of the ECM in the in vitro $3 \mathrm{D}$ cultures.

\section{TEM Analysis of 3D Structures}

Using TEM, we examined three timepoints during osteoblast 3D culture, at 15, 22, and 48 days. This method of analysis does not permit easy identification of the region within the sample where the inspection is directed, but proved very useful for the identification of morphological features of different cell types, identification of specific structures, and demonstration of the organization of collagen and deposition of mineral in the extracellular matrix. The 3D structure analyzed after 15 days of culture revealed closely adherent cells with active nuclei, rich in euchromatin, and with a generously sized cytoplasm organelle compartment. A majority of preosteoblasts displaying pools of glycogen in their cytoplasm were detected at this timepoint and the ECM was sparse, containing only a few narrow-diameter collagen fibrils and associated ground substance material.

No mature osteoblasts or osteocytes were detected; other cells were fibroblast-like (not shown). Rare mitotic (Figure 3d) and apoptotic figures were detected. No aggregation of mineral was observed. After 22 days of culture, the 3D structures were more heterogeneous and contained a variety of cell types. Osteoblasts were more widely dispersed compared with the closely adherent cell aggregations present at the earlier timepoint. Typical preosteoblasts, elongated in shape and containing large pools of glycogen, were sometimes closely adjacent. Cells exhibiting the characteristics of mature osteoblasts were frequent, with prominent rough endoplasmic reticulum and Golgi elements together with moderate numbers of mitochondria and an occasional lysosome. The ECM organization contained extensive areas wherein interwoven fibrils of collagen were detected. The mature osteoblasts resided in a mineralizing collagenous ECM, which had deposits of needle-shaped crystals, characteristic of the mineralizing osteoid of bone in vivo. Gap junctions were present between some adjacent osteoblasts (not shown). Many mature osteoblasts (Figure 3a) and cells displaying formative osteocyte characteristics (Figure 3b) were present in the 3D structures after 47 days in culture. The ECM was composed of interwoven collagenous fibrils and ground substance and displayed extensive mineralization associated with the presence of needle-shaped crystals, which expanded into nodular arrangements. These crys- 

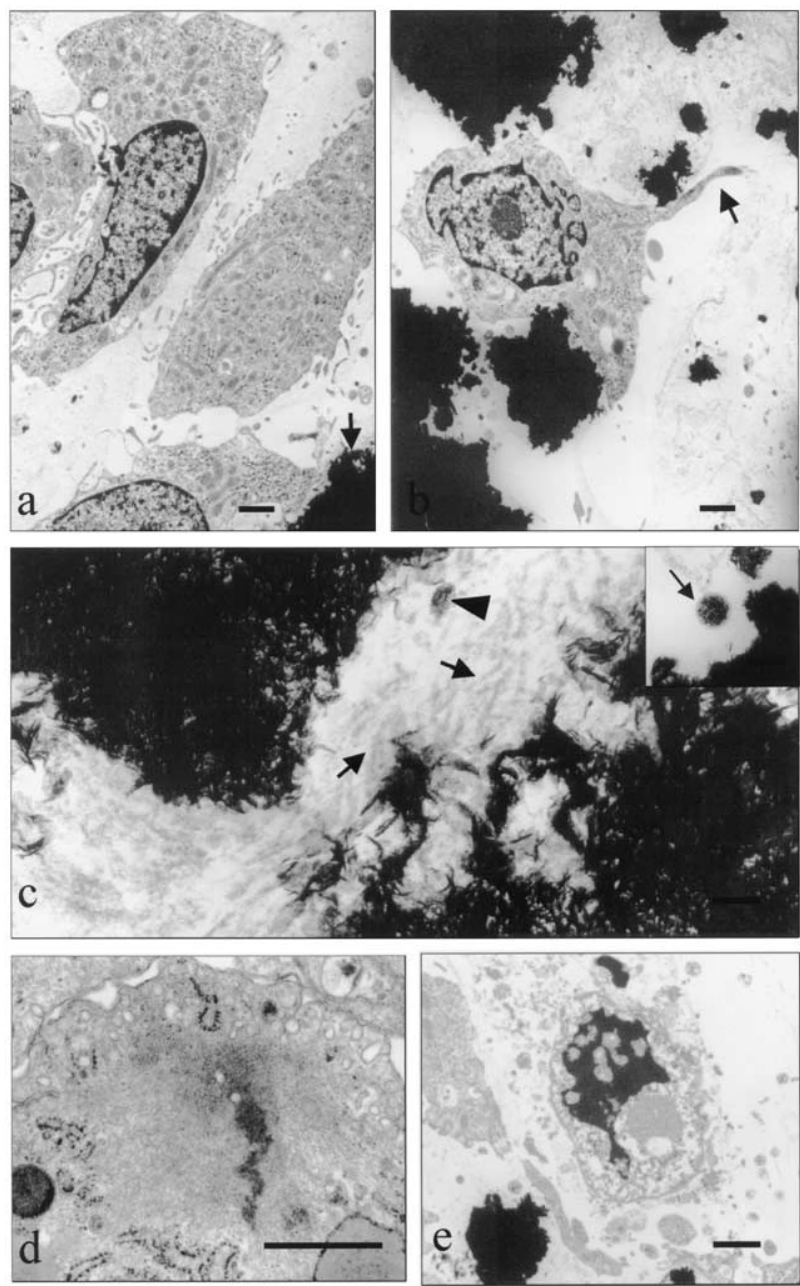

Figure 3. TEM analysis of $3 \mathrm{D}$ culture. 3D cultures after 15 days of differentiation in vitro (d) and after 47 days of differentiation in vitro (a-c,e). (a) Portions of several osteoblasts; note mineralizing matrix (arrow). (b) A formative osteocyte in a mineralized matrix; note the elongated cell process (arrow). (c) Aggregations of needle-shaped mineral crystals in the ECM are associated with densely interwoven collagen fibrils (arrows), ruptured matrix vesicle (arrowhead), and mineralizing matrix vesicle (inset, arrow) adjacent to a mineralizing region. (d) Portion of a mitotic cell apparently in metaphase; note the spindle apparatus centrally and the classical peripheral distribution of cell organelles. (e) A cell displaying an apoptotic nucleus. Scale bars: (a), (b), (d), (e) $1 \mu \mathrm{m}$; (c), (inset) $100 \mathrm{~nm}$.

tals were grouped into regions of more extensive mineralization (Figure $3 \mathrm{~b}, \mathrm{c}$ ). There was also evidence of discrete mineralization foci being initiated by matrix vesicles as demonstrated by membrane-bound (Figure 3c, inset) and ruptured (Figure 3c) matrix vesicles. In the ossicles dead cells were rarely detected, with highly condensed nuclear chromatin (not shown), and dying cells with apoptotic nuclei (Figure 3e).

\section{Implantation in Nude Mice of $3 D$ Structures Cultured for Different Time Lengths In Vitro}

We grafted the 3D structures subcutaneously into the nude mice. The 3D structures cultured in vitro for 15 and 36 days were grafted, and recovered 20 days later in each of two different experiments with osteoblasts derived from female patients, 73 and 42 years old, respectively. Four 3D structures for each timepoint were implanted, each into a different mouse. Upon retrieval, all explants showed a peripheral multilayer of cells embedded in a collagenous matrix, as detected by Azan-Mallory and Van Gieson histochemistry techniques (not shown), and were consistently thicker in the implants of the 3D structures cultured in vitro for the shorter time duration. All explants also displayed a core region, with sparser cells within a collagenous ECM. This region was more conspicuous in size in the implants made after the longer permanence in vitro of the 3D structures. All explants were negative for AP expression (not shown), consistent with loss of this early osteogenic marker in vitro with increasing culture time. Mineralization was revealed by Von Kossa staining (Figure 4a,b) in structures implanted after both duration of culture in vitro, and higher in intensity in those derived from 3D structures implanted after the longer duration in vitro. Mineral deposition was located most often around or near cavities in the ECM (Figure 4c,d) and the edges of these cavities showed cells embedded in a collagenous ECM (Figure 4f,h). All explants were vascularized by invasion of endothelial cells from the host (Figure 4e,g,h). Indeed, vessels containing blood cells were evident in the peripheral multilayer, as well as in the core region of the implant, as early as 14 days after implantation (not shown).

Testing for tartrate-resistant acid phosphatase (TRAP) expression by histochemistry did not reveal multinucleated positive cells and showed, at most, one mononuclear positive cell per section, regardless of the duration of their previous culture in vitro (not shown), thus excluding that relevant invasion by osteoclasts had occurred during the 20 day period of the in vivo experiment.

\section{Discussion}

We have reported a novel procedure for obtaining three-dimensional cultures, by preserving, to the extent possible, the physiological microenvironment produced by the osteoblasts themselves as to the quality and quantity of the endogenous pericellular and extracellular components and with regard to cell-cell and cell-ECM relationships.

The 3D cultures thus obtained showed the initial establishment of close cell-cell relationships (tight junctions) and expression of AP, which declined with time. Bone sialoprotein, collagen type I, osteonectin, fibronectin, decorin, and osteopontin were detected throughout the culture, whereas expression of osteocalcin was restricted to the longest culture time. The sequential morphological changes with time in culture, documented by TEM, indicated phenotypic maturation from early to mature osteoblasts and to cells with the features of formative osteocytes.

The ECM accumulating between cells became enriched in interwoven collagen fibrils with associated needle-shaped crystals characteristic of bone, which also involved membrane-bound matrix vesicles. Calcium incorporation proceeded to levels tenfold higher than in the corresponding $2 \mathrm{D}$ cultures and mineralization was confirmed by histochemistry. Thus, in vitro 3D cultures of adult human osteoblasts are capable of full differentiation up to mineral deposition in the absence of exogenous osteotropic agents, supporting that the interactions among osteoblasts and of those with their pericellular environment are key factors for the progression of differentiation in human postpubertal osteoblasts. Similar conclusions were recently reached in a study in which adult human osteoblasts were induced by treatment with TGF- $\beta$ to form aggregates, which mineralized in vitro. $^{24}$

In $3 \mathrm{D}$ cultures osteoblasts also preserve their potential for 


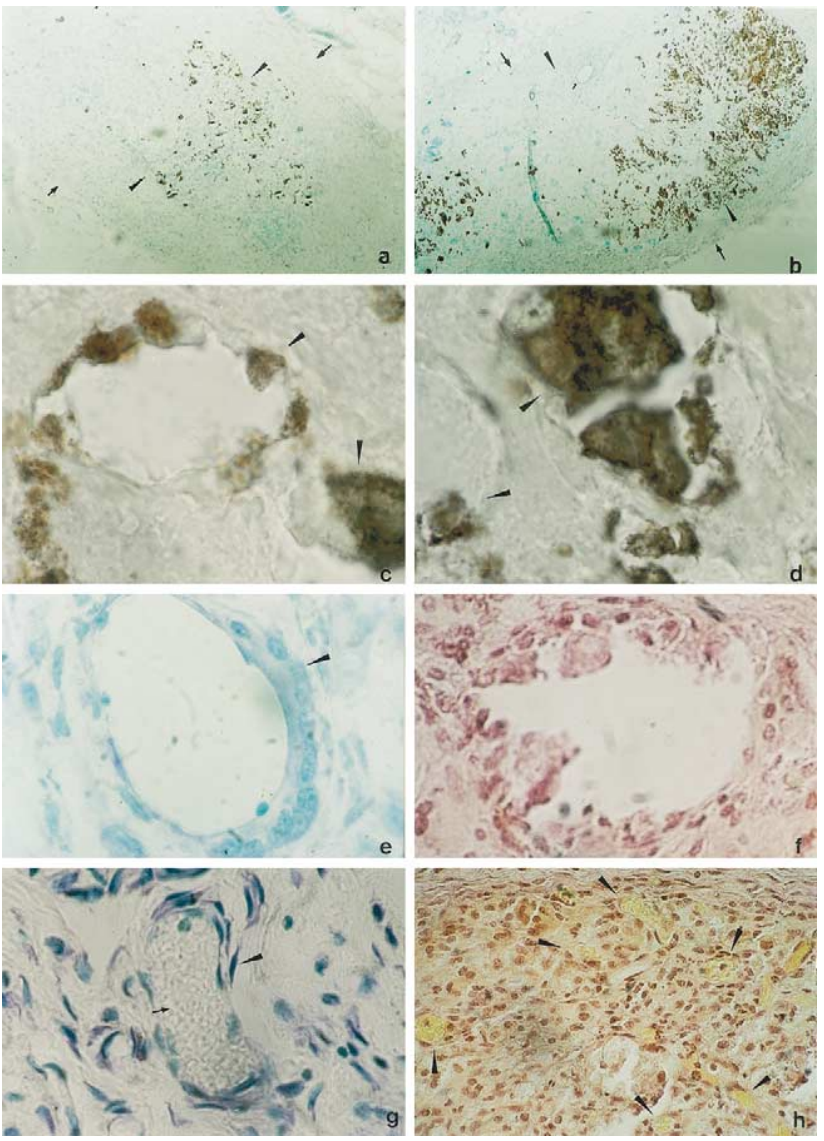

Figure 4. Mineralization and vascularization in the explants of 3D cultures. Mineralization revealed by Von Kossa staining in explants of 3D cultures implanted after 15 days [(a) and (c)] and 36 days [(b) and (d)] of culture in vitro and 20 days in vivo. Original magnification: (a), (b) $\times 200$; (c), (d) $\times 2000$. Mineralized regions around cavities $[(c)$ and (d)] and within matrix [bottom right in (c)] are shown. Regions corresponding to those wherein mineral is deposited are also shown, and stained with Giemsa (e) and Van Gieson (f), demonstrating the presence of cells on the edges of cavities and showing the collagenous ECM (f) (original magnification: $\times 200$ ). Vessels (arrowheads), including red cells (arrows), are shown in explants of 3D cultures implanted after 15 days [(g) original magnification: $\times 800]$ and 36 days $[(\mathrm{h})$ original magnification $\times 400$ ] of culture in vitro and 20 days in vivo stained with Giemsa and Van Gieson, respectively.

growth and differentiation for $>120$ days, which was further supported by the detection of mitotic figures by TEM. Occasional cell death also occurred, at the latest timepoint, displayed as nuclei with condensed chromatin by Giemsa staining (not shown), and as apoptotic cells by TEM. It is possible that cell proliferation and cell death occur in different regions of the $3 \mathrm{D}$ structure during in vitro culture. This could be determined by differences in cell-cell and/or cell-ECM relationships and/or by the relationship with the surrounding environment. Growth may be continuing mostly at the periphery, whereas, at late timepoints, death was detected in the most internal region. (We do not know if this is programmed, caused by hypoxia, or initiated by lack of sufficient nutrient flux.)

The fact that 3D cultures from adult-derived osteoblasts support continuing cell differentiation and proliferation in vitro coincides with the fact that proliferation and differentiation is maintained in osteoblasts from patients and from mice with secondary osteopenias. ${ }^{8}$
Culturing 3D in vitro adult-derived human osteoblasts in the endogenous ECM is the first method reported to date whereby complete osteogenic progression is obtained without the addition of exogenous substrates, growth factors, or hormones. It provides a model system for studies in vitro of the later phase of osteogenesis in human cells where is possible to test the effects of drugs, growth factors, and hormones on mature osteoblasts and osteocyte-like cells. This culture system is also suitable for study of the osteogenic and growth potentials of bone cells derived from patients affected by genetic and/or metabolic pathologies of bone.

The 3D cultures are presently implemented in our laboratory as a tool for establishing in vitro cocultures with different cell types of the in vivo bone microenvironment. These are being developed with an aim to study the induction of osteoclastic differentiation and endothelial morphogenesis ${ }^{36}$ (C. Biassoni, Thesis, 2001), and the proliferation of cancer cells with preferential homing to bone in the osteogenic microenvironment (D. Palmieri et al., manuscript in preparation).

The experiments we performed by implanting in vivo 3D structures cultured in vitro were directed primarily to establish if these could continue differentiation in the ectopic location. We therefore implanted 3D structures that were at an earlier stage of maturation in vitro (15 days, unmineralized) and at a later stage (36 days, wherein mineral deposition had already started), and examined them after 20 days of permanence in nude mice. Once explanted, cultures of both ages in vitro (and consistently in all 12 samples from the two experiments) showed a peripheral collar consisting of a multilayer of cells embedded in a collagen-rich matrix and an internal core region, with cells embedded in abundant collagen-rich and mineralizing ECM. Both compartments, analyzed by immunohistochemistry, were stained by a bovine/human specific antibody against osteonectin and therefore were of human origin. The extent of peripheral collar was inversely proportional to the age previously achieved in vitro by the implanted 3D structure, suggesting it might derive from the outgrowth of cells, which may be more extensive for "younger" implants. Although, in principle, the presence of different regions could also derive from differences in nutrient supply, this it is unlikely because another feature consistently found in all explants after 14 days in vivo was a diffuse network of vessels, containing blood cells, and present throughout the implant. It cannot be ruled out that interactions within the subcutaneous environment might be also instructive for the organization of implants in these two layers. The viability of the implanted 3D structures was maintained throughout the 20 days of the experiments. Terminal in situ nick-translation experiments showed less than one stained cell per section (not shown).

Study of the expression of osteogenic markers showed that progression of differentiation occurred during the in vivo culture. Although AP expression was not detected in explants, according to its decline in the late phases of osteogenic progression, mineralization was always detected. All explants (six of six) of 3D structures either when implanted before being mineralized in vitro (15 days of culture) or when implanted after onset of mineralization in vitro ( 36 days of culture) were more heavily stained by Von Kossa at the time of explant, 20 days later, than when implanted. Three-D structures implanted after 36 days of active in vitro were more heavily stained by Von Kossa than those implanted after 15 days of culture in vitro, at retrieval, after 20 days in vivo. Mineral in these explants was deposed most often around and intruding into cavities of the ECM. When successive sections to those stained by Von Kossa were inspected, we detected cells embedded in the collagenous matrix on the edges of these cavities. The cavities themselves may have resulted from proteolytic processing of the ECM by osteoblasts 
(no polynucleate osteoclasts were detectable by TRAP; see later), possibly accompanied by mineralization at the edges. Nonetheless, it cannot be ruled out that the cavities derived from a trivial event during sectioning of the 3D structure with different internal resistance to the blade. If this were the case, it would not subtract to the observation that, in adjacency to the regions where mineral is more heavily deposited, cells (and matrix vescicles illustrated by TEM) are present. Both in 3D structures in vitro and in vivo, the mineralization involved discrete areas. No clear marrow cavity and bone marrow formation was observed in the 20 days of culture in vivo. A mineralization front comparable to that observed in vivo or in 3D structures obtained by seeding cells on a scaffolding was absent in the 3D structures described here, and is, analogous to its absence in 3D cultures, produced in the presence of TGF- $\beta$. Possibly this is related to the fact that both of these procedures involved the formation of a compact structure of cells and ECM. No mineral deposition was detected in the peripheral collar of the implants, and there was no osteinduction of adjacent host tissues. After 20 days in vivo, only occasional TRAP-positive mononucleated cells were identified as macrophages by TEM (not shown), whereas no TRAP-positive polynucleate cells were detected in explants, suggesting that osteoclastic maturation of host monocytes is not promoted in this time frame or under these conditions.

As mentioned earlier, pervasive vascularization of the implants was observed already after 14 days in vivo. Vascularization in the implants is in agreement with the production of the C-terminal trimer of procollagen type I by mature osteoblasts (Manduca, unpublished data), which is a chemoattractant for endothelial cells in vitro. ${ }^{36}$

In summary, osteogenesis in 3D structures also progresses in vivo and the implants are a tool to assess the effects of systemic factors and specific treatments potentially relevant in osteogenesis and by changing the time of implantation, to each stage of the osteogenic progression. Among the most obvious advantages of the present system of implants in vivo of $3 \mathrm{D}$ structures is the high rate of success in progression throughout differentiation of implants (12 of 12), independent of donor age and gender (25-73 years of age). The 3D culture also offers the novel possibility to implant structures at different and controlled stages of osteogenic progression. Implants of 3D structures containing osteoblasts and other cell types, presently under study in our laboratory, permit investigation of the molecular interactions between the different cell types found in the bone compartment and of the osteogenic environment with tumor-derived cells, homing preferentially to bone.

Acknowledgments: This study was Funded by Ateneo, Progetto Nazionale MURST, and Biotecnologie CNR (to Dr. Manduca) and the Queen's University of Belfast (to Dr. Dickson)

\section{References}

1. Aubin, J. E., Liu, F., Malaval, L., and Gupta, A. K. Osteoblast and chondroblast differentiation. Bone 17(Suppl.):77S-83S; 1995.

2. Bab, I., Ashton, B. A., Syftestad, G. T., and Owen, M. E. Assessment of an in vivo diffusion chamber method as a quantitative assay for osteogenesis. Calcif Tissue Int 36:77-82; 1984.

3. Bennet, J. H., Joyner, C. J., Triffitt, J. T., and Owen, M. E. Adipocytic cells cultured from marrow have osteogenic potential. J Cell Sci 99:131-139; 1991.

4. Bidwell, J. P., Alvarez, M., Feister, H., Onyia, J., and Hock, J. Nuclear matrix proteins and osteoblast gene expression. J Bone Miner Res 13:155-167; 1998.

5. Boynton, E., Aubin, J., Gross, A., Hozumi, N., and Sandhu, J. Human osteoblasts survive and deposit new bone when human bone is implanted in SCID mouse. Bone 18:321-326; 1996.

6. Bowman, N. N., Donahue, H. J., and Ehrlich, H. P. Gap junctional intercellular communication contributes to the contraction of rat osteoblast populated collagen lattices. J Bone Miner Res 13:1700-1706; 1998.

7. Casser-Bette, M., Murray, A. B., Closs, E. I., Erfle, V., and Schmidt, J. Bone fromation by osteoblast-like cells in a three-dimensional cell culture. Calcif Tissue Int 46:46-56; 1990.

8. D'Ippolito, G., Schiller, P. C., Ricordi, C., Roos, B. A., and Howard, G. A. Age-related osteogenic potential of mesenchymal stromal stem cells from human vertebral bone marrow. J Bone Miner Res 14:1115-1122; 1999.

9. Donahue, H. J., McLeod, K. J., Rubin, C. T., Andersen, J., Grine, E. A., Hertzberg, E. L., and Brink, P. R. Cell-to-cell communication in osteoblastic networks: Cell line-dependent hormonal regulation of gap junction function. J Bone Miner Res 10:881-889; 1995.

10. Ernst, M. and Froesch, E. R. Osteoblastlike cells in a serum-free methylcellulose medium form colonies: Effects of insulin and insulin-like growth factor I. Calcif Tissue Int 40:27-34; 1987.

11. Filanti, C., Dickson, G. R., Di Martino, D., Ulivi, V., Sanguineti, C., Romano, P., Palermo, C., and Manduca, P. The expression of metalloproteinases ( -2 , $-9,-14)$ and of tissue inhibitors $(-1,-2)$ is developmentally modulated during osteogenesis in vitro, the mature osteoblastic phenotype expressing metalloproteinase-14. J Bone Miner Res 15:2154-2168; 2000.

12. Fisher, L. W., Hawkins, G. R., Tuross, N., and Termine, J. D. Purification and partial characterization of small proteoglycans I and II, bone sialoproteins I and II, and osteonectin from the mineral compartment of developing human bone. J Biol Chem 262:9702-9708; 1987

13. Fisher, L. W., Termine, J. D., and Young, M. F. Deduced protein sequence of bone small proteoglycan I (biglycan) shows homology with proteoglycan II (decorin) and several non connective tissue proteins in a variety of species. J Biol Chem 264:4571-4576; 1989.

14. Gehron Robey, P. and Termine, J. D. Human bone cells in vitro. Calcif Tissue Int 37:453-456; 1985 .

15. Gerstenfeld, L. C., Uporova, T., Schmidt, J., Strauss, P. G., Shih, S. D., Huang, L. F, Gundberg, C., Mizuno, S., and Glowacki, J. Osteogenic potential of murine osteosarcoma cells: Comparison of bone-specific gene expression in in vitro and in vivo conditions. Lab Invest 74:895-906; 1996.

16. Gundle, R., Joyner, C. J., and Triffitt, J. T. Human bone tissue formation in diffusion chamber culture in vivo by bone-derived cells and marrow stromal fibroblastic cells. Bone 16:597-601; 1995

17. Gurdon, J. B., Lemaire, P., and Kato, K. Community effects and related phenomena in development Cell 75:831-834; 1993.

18. Hara, A., Ikeda, T., Nomura, S., Yagita, H., Okumura, K., and Yamauchi, Y. In vivo implantation of human osteosarcoma cells in nude mice induces bones with human-derived osteoblasts and mouse-derived osteocytes. Lab Invest 75:707-717; 1996

19. Hill, P. A., Tumbler, A., and Meikle, M. C. Multiple extracellular signals promote osteoblast survival and apoptosis. Endocrinology 138:3849-3858; 1997.

20. Hott, M., Noel, B., Bernache, A., Assolant, D., Rey, C., and Marie, P. J. Proliferation and differentiation of human trabecular osteoblastic cells on hydroxyapatite. J Biomed Mater Res 4:508-516; 1997.

21. Ingber, D. E. Tensegrity: The architectural basis of cellular mechanotransduction. Ann Rev Physiol 59:575-599; 1997.

22. Ishaug-Riley, S., Crane-Kruger, G. M., Yaszemski, M. J., and Mikos, A. G. Three-dimensional culture of rat calvarial osteoblasts in porous biodegradable polymers. Biomaterials 19:1405-1412; 1998.

23. Jimenez, M. J. G., Balbin, M., Lopez, J. M., Alvarez, J., Komori, T., and Lopez-Otin, C. Collagenase 3 is a target of Cbfa1, a transcription factor of the runt gene family involved in bone formation. Mol Cell Biol 19:4431-4442; 1999.

24. Kale, S., Biermann, S., Edwards, C., Tarnowski, C., Morris, M., and Long, M. W., Three-dimensional cellular development is essential for ex vivo formation of human bone. Nat Biotechnol 18:954-958; 2000.

25. Krebsbach, P. H., Kuznetsov, S. A., Satomura, K., Emmons, R. V. B., Rowe, D. W., Gerhon Robey, P., Comparison of osteogenesis by transplanted mouse and human marrow stromal fibroblasts. Transplantation 63:1059-1069; 1997.

26. Kuznetsov, S. A., Krebsbach, P. H., Satomura, K., Kerr, J., Riminucci, M., Benayahu, D., and Gerhon Robey, P., Single-colony derived strains of human marrow stromal fibroblasts form bone after transplantation in vivo. J Bone Miner Res 12:1335-1347; 1997.

27. Lynch, M. P., Stein, J. L., Stein, G. S., and Lian, J. B. The influence of type I collagen on the development and maintenance of the osteoblast phenotype in primary and passaged rat calvarial osteoblasts: Modification of expression of genes supporting cell growth, adhesion, and extracellular matrix mineralization. Exp Cell Res 216:35-45; 1995. 
28. Manduca, P., Descalzi Cancedda, F., and Cancedda, R. Chondrogenic differentiation in chick embryo osteoblast cultures. Eur J Cell Biol 57:193-201; 1992.

29. Manduca, P., Sanguineti, C., Pistone, M., Boccignone, E., Sanguineti, F., Santolini, F., and Federici, A. Differential expression of alkaline phopshatase in clones of human osteoblast like cells. J Bone Miner Res 8:291-300; 1993.

30. Mizuno, M., Shindo, M., Kobayashi, D., Tsuruga, E., Amemiya, A., and Kuboki, Y. Osteogenesis by bone marrow stromal cells maintained on type I collagen matrix gels in vivo. Bone 20:101-107; 1997.

31. Moursi, A. M., Damsky, C. H., Lull, J., Zimmerman, D., Doty, S. B., Aota, S., and Globus, R. K. Fibronectin regulates calvarial osteoblast differentiation. J Cell Sci 109:1369-1380; 1996.

32. Nishimoto, S. K., Stryker, W. F., and Nimni, M. E. Calcification of osteoblastlike rat osteosarcoma cells in agarose suspension cultures. Calcif Tissue Int 41:274-280; 1987.

33. Nuttall, M. E., Patton, A. J., Olivera, D., Nadeau, D. P., and Gowen, M. Human trabecular bone cells are able to express both osteoblastic and adipocytic phenotype: Implications for osteopenic disorders. J Bone Miner Res 13:371382; 1998.

34. Oreffo, R. O., Driessens, F. C., Planell, J. A., and Triffitt, J. T. Growth and differentiation of human bone marrow osteoprogenitors on novel calcium phosphate cements. Biomaterials 20:1845-54; 1998.

35. Owen, T. A., Aronow, M., Shalhoub, V., Barone, L. M., Wilming, L., Tassinari, M. S., Kennedy, M. B., Pockwinse, S., Lian, J. B., and Stein, G. S.
Progressive development of the rat osteoblast phenotype in vitro: Reciprocal relationship in expression of genes associated with osteoblast proliferation and differentiation during formation of the bone extracellular matrix. J Cell Physiol 143:420-430; 1990.

36. Palmieri, D., Camardella, L., Ulivi, V., Guasco, G., and Manduca, P. Trimer carboxyl pro-peptide of collagen I produced by mature osteoblasts is chemotactic for endothelial cells. J Biol Chem 275:32658-32663; 2000.

37. Saad, B., Matter, S., Ciardelli, G., Uhlschmid, G. K., Welti, M., Neuenschwander, P., and Suter, U. W. Interactions of osteoblasts and macrophages with the biodegradable and highly porous polyester urethane foam and its degradation products. J Biomed Mater Res 32:355-366; 1996.

38. Spurr, A. R. A low viscosity epoxy resin embedding medium for electron microscopy. J Ultrastruct Res 26:31-35; 1969.

39. Sudo, H., Kodama, H., Amagai, Y., Itakura, Y., and Yamamoto, S. Mineralized tissue formation by MC3T3-E1 osteogenic cells embedded in three-dimensional gel matrix. In: Ali, S. Y., Ed. Cell-mediated Calcification and Matrix Vescicles. Amsterdam: Elsevier; 1986; 291-296.

Date Received: July 3, 2001

Date Revised: December 21, 2001

Date Accepted: December 21, 200 